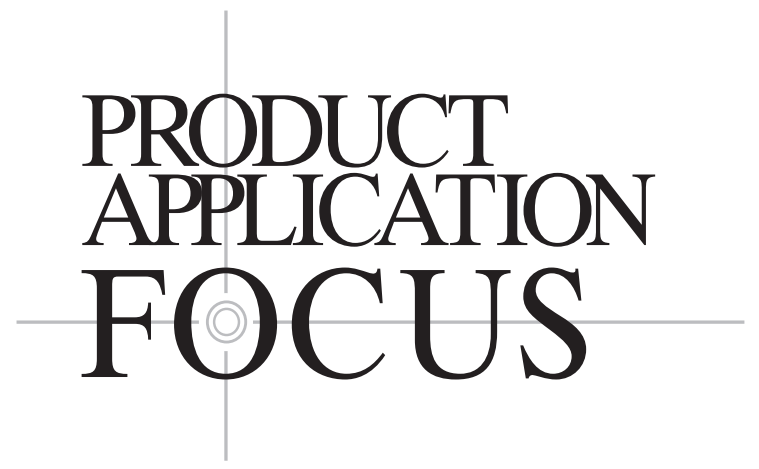

... a forum for manufacturers to describe the current and potential applications of new research instruments or products.

\title{
Automated High-Throughput Probe Production for DNA Microarray Analysis
}

\author{
P.R. Hoyt, L. Tack ${ }^{1}$, B.H. Jones, J. Van Dinther ${ }^{1}$, S. Staat ${ }^{1}$, and M.J. Doktycz \\ Oak Ridge National Laboratory, Oak Ridge, TN, and ${ }^{1}$ PerkinElmer Life Sciences, \\ Downers Grove, IL, USA
}

BioTechniques 34:402-407 (February 2003)

\begin{abstract}
DNA microarrays have become an established tool for gene expression profiling. Construction of these microarrays using immobilized cDNAs is a common experimental strategy. However, this is extremely laborious, requiring the preparation of hundreds or thousands of cDNA probes. To minimize this initial bottleneck, we developed a comprehensive high-throughput robotic system to prepare DNA probes suitable for microarray analysis with minimal user intervention. We describe an automated system using the MultiPROBE ${ }^{\circledR}$ Nucleic Acid Purification Workstation to provide the liquid handling and other functions needed to optimize this process. We were able to carry out fully automated plasmid cDNA isolation, PCR assay setup, and PCR purification and also to direct the characterization and tracking of DNA probes during processing. Protocols began with the initial preparation of a plasmid DNA archive of bacterial stocks in parallel 96-well plates (192 samples/run) and continued through to the dilution and reformatting of chip-ready DNA probes in 384-well format. These and other probe production procedures and additional instrument systems were used to process fully a set of mouse cDNA clones that were then validated by differential gene expression analysis.
\end{abstract}

\section{INTRODUCTION}

Microarray technology allows the analysis of expression levels for thousands of genes in a miniaturized parallel format. Commonly, PCR-amplified cDNA clone inserts are spotted onto glass microscope slides. This format is now accessible to large communities of users with the availability of spotting and array analyzing instruments and with core facilities becoming routine in both academic and industrial settings.

The "front-end" sample preparation stage for chip construction involves more time and effort than later steps (e.g., hybridization). Typical cDNA libraries contain 10000 or more unique clones and require multiple processing steps before spotting. Additional quality-control measures to assure array integrity (i.e., gel electrophoresis, sample quantitation, and sample tracking) are also necessary.

While several automated platforms for plasmid DNA isolation have been reported $(1,4,5,7-11,14)$, many are dedicated to PCR and sequencing applications and have limited utility for high-throughput DNA array production. This can result in purchasing prepared clones or resorting to tedious, errorprone manual processes to produce array reagents. We have identified a robotic system with the flexibility to carry out a comprehensive set of fully automated array probe production functions. This system, based around the Packard-brand MultiPROBE $^{\circledR}$ II Nucleic Acid Purification Workstation (Perkin-Elmer Life Sciences, Downers Grove, IL, USA), processes samples using a variety of protocols. It accommodates walk-away DNA probe processing at both low- or highthroughput levels. We describe automation of microarray probe preparation using this system to both improve throughput and increase probe quality. By reducing purchases and laboratory-space commitments to a single instrument, economy of production is also maximized. 


\section{MATERIALS AND METHODS}

\section{Reagents and Supplies}

The NIA $15 \mathrm{~K}$ clone set (17) was purchased from Incyte Genomics (Palo Alto, CA, USA) or Research Genetics (Huntsville, AL, USA). QIAprep ${ }^{\circledR} 96$ Turbo Miniprep Kits and QIAquick ${ }^{\mathrm{TM}} 96$ PCR Purification Kits were purchased from Qiagen (Valencia, CA, USA). PCR kits were from PerkinElmer/Roche (Branchburg, NJ, USA) and Promega (Madison, WI, USA).

Conductive pipet tips were purchased from PerkinElmer Life Sciences (Boston, MA, USA) or Molecular BioProducts (San Diego, CA, USA). PicoGreen ${ }^{\circledR}$ dsDNA quantitation reagent was from Molecular Probes (Eugene, OR, USA). Probe labeling reagents were from Sigma (St. Louis, MO, USA). CMT-GAPS ${ }^{\mathrm{TM}}$ amino-silane coated microscope slides were from Corning (Corning, NY, USA). Standard molecular biology protocols (12) were followed.

\section{Probe Preparation Instrumentation}

The core liquid handling workstation used for probe processing was the Packard Nucleic Acid Purification Workstation consisting of MultiPROBE II HT EX with Gripper Integration Platform, WinPREP ${ }^{\circledR}$ software, PVM Vacuum System, DPC Micromix 5 Shaker, and Nucleic Acid Extraction Application Option $(15,16)$. For array construction, we used the VIRTEK Chip Writer (model SDDC-2; Ontario, Canada) fitted with four Arrayit ${ }^{\mathrm{TM}}$ Stealth MicroSpotting pins that dispense $0.6 \mathrm{~nL} /$ touch (Telechem International, Sunnyvale, CA, USA) for spotting microarrays and a Packard ScanArray ${ }^{\circledR}$ 4000XL microarray confocal laser scanner for imaging.

Other equipment included a Forma model 4518 Shaking Incubator (Marietta, OH, USA), Sorvall (Newtown, CT, USA) RC5B centrifuge with SH3000 rotor, PerkinElmer/Roche GeneAmp ${ }^{\circledR} 9600$ thermal cycler, and a PerkinElmer HTS 7000 Plus Bioassay Plate Reader. Gel electrophoresis was performed using an Owl Separation Systems model D3-14 unit (Portsmouth, NH, USA).

\section{Plasmid Preparation}

Plasmid stocks were replicated using a 96-pin tool capable of 25- $\mu \mathrm{L}$ liquid transfers (V\&P Scientific, San Diego, CA, USA) into 96-well deep well growth plates (Whatman, Clifton, NJ, USA) containing $1.5 \mathrm{~mL}$ enriched $2 \times$ YT bacterial media (17). Bacteria were grown $16 \mathrm{~h}$ at $37^{\circ} \mathrm{C}$ with shaking $(250 \mathrm{rpm})$. Subsequently, two plates of bacterial cells were pelleted by centrifugation at $5000 \times g$ for $15 \mathrm{~min}$. Plates were inverted, blotted to remove excess media, and placed on the MultiPROBE deck before starting an automated protocol.

Automated plasmid DNA purification was carried out using two PVM manifolds placed on the MultiPROBE deck, a custom WinPREP Nucleic Acid Extraction template, and the filter plates and reagents provided with the QIAprep kit (15). The nucleic acid extraction protocol initiated with resuspension of bacterial cell pellets in two growth plates and ended with elution of purified plasmid DNA samples $(100 \mu \mathrm{L})$ into two 96-well microplates (Corning Costar, Cambridge, MA, USA) placed in the manifold base.

\section{PCR Product Preparation}

PCRs used for microarray probe production contained $1 \times$ reaction buffer, $2 \mathrm{mM} \mathrm{MgCl}_{2}, 2 \mathrm{mM}$ each dNTP, $0.2 \mathrm{U} / \mu \mathrm{L}$ Taq DNA polymerase, $2 \mathrm{ng} / \mu \mathrm{L}$ each of primers $\left(5^{\prime}\right.$-GTTTTCCCAGTCACGACGTTG-3' and 5'-TGAGCGGATAACAATTTCACACAG-3'; Integrated DNA Technologies, Coralville, IA, USA) (17), and approximately $4 \mathrm{ng} / \mu \mathrm{L}$ plasmid DNA per $50 \mu \mathrm{L}$ reaction. PCR conditions were $95^{\circ} \mathrm{C}$ for 5 min, followed by 40 cycles of $94^{\circ} \mathrm{C}$ for $30 \mathrm{~s}, 54^{\circ} \mathrm{C}$ for $30 \mathrm{~s}$, and $73^{\circ} \mathrm{C}$ for $3.5 \mathrm{~min}$, finished with $7 \mathrm{~min}$ at $72^{\circ} \mathrm{C}$. The MultiPROBE II assembled the reactions by aliquoting template DNA and PCR master mixture directly into 96-well PCR plates. The plates were positioned inside a round-bottomed 96-well microplate placed on a plate support tile. Samples plates were removed to the thermal cycler for amplification and returned to the deck for further processing.

PCR products in two 96-well plates were automatically purified using the MultiPROBE workstation, a custom WinPREP PCR Cleanup template, and the filter plates and reagents provided with the QIAquick kit. The protocol ended with elution $(100 \mu \mathrm{L} /$ well $)$ of purified PCR fragments into each of two 96 -well microplates. PCR products were dried overnight at $50^{\circ} \mathrm{C}$ and uniformly resuspended in $25-30 \mu \mathrm{L} 3 \times$ SSC buffer depending on DNA quantitation results (not shown) to prepare approximately $150 \mu \mathrm{g} / \mathrm{mL}$ solution, as recommended for spotting by the slide manufacturer (Corning). The sample concentrations were not normalized. These resuspension volumes resulted in sample concentrations that were equal or greater than $150 \mathrm{ng} / \mu \mathrm{L}$ for $95 \%$ of the samples. The MultiPROBE then redistributed the DNA samples into 384well polypropylene microplates (Whatman) before spotting onto microarray slides. Using $30 \mu \mathrm{L}$ volume for the samples in a spotting plate, and accepting that $0.6 \mathrm{~nL}$ is dispensed per touch, a theoretical maximum of 50000 spots per sample is possible with this preparation scheme. Spotting each sample in triplicate would reduce the maximum theoretical number of arrays possible to 16666 .

\section{Gel Electrophoresis}

Electrophoretic analysis of both plasmid and PCR-amplified DNA was carried out on the MultiPROBE deck. For automated loading, a $1.2 \%$ submerged agarose gel in TAE buffer (12) was cast using a modified Owl electrophoresis unit. Lucite ${ }^{\mathrm{TM}}$ retainers, placed inside the buffer chamber, held the gel plate precisely in the middle during loading. A 1.5-mm-wide spacer was placed between the gel tray and the buffer chamber wall. The well combs were manually positioned at the top-left registration position during pouring. The entire gel unit was mapped using WinPREP software, creating a new labware definition. Typically, $3 \mu \mathrm{L}$ plasmid or PCR-amplified DNA were mixed with $15 \mu \mathrm{L} 1.5 \times$ DNA loading buffer (12) pre-aliquoted in a separate 96-well plate. Using the same pipet tip, the mixed samples were loaded directly into the submerged gel wells. 


\section{DNA Quantitation}

Automated fluorescence-based DNA quantitation was performed using the MultiPROBE. Plasmid DNA or PCR product $(3 \mu \mathrm{L})$ was added to $497 \mu \mathrm{L}$ TE $(10 \mathrm{mM}$ Tris, $1 \mathrm{mM}$ EDTA, pH 7.4). The diluted DNA $(50 \mu \mathrm{L})$ was mixed with $50 \mu \mathrm{L}$ of a 1:200 dilution of PicoGreen reagent in a 96-well black flat-bottom Dynatech Microfluor ${ }^{\circledR} 1$ plate (Thermo Labsystems, Helsinki, Finland). A standard concentration plot was generated under identical conditions with known DNA concentrations. Plates were read (fluorescence mode: excitation wavelength, $485 \mathrm{~nm}$; emission wavelength, 535 $\mathrm{nm}$ ) in the HTS 7000 plate reader. The raw data were exported to a Microsoft ${ }^{\circledR}$ Excel $^{\circledR}$ spreadsheet linked with the clone database for DNA concentration calculation and normalization.

\section{Sample Tracking}

Clonally derived samples were tracked through purification steps using the Microsoft Access ${ }^{\mathrm{TM}}$ database integrated with WinPREP software. We wrote custom processes in Microsoft Access to automate tracking that used both sample positional information and plate names assigned using WinPREP. A clone "master" list was generated in Excel-compatible format to link the plate name, row, and column information to the clone identity, plus additional information. Selected columns were also extracted into a new CloneName $(\mathrm{CN})$ text-file derived from positional information accumulated during redistribution into 384-well plates for spotting. The $\mathrm{CN}$ text-file was input into CloneTracker ${ }^{\mathrm{TM}}$ (BioDiscovery, Marina Del Rey, CA, USA) software for subsequent tracking of the clone positions and gene information while spotting and analyzing microarrays. CloneTracker software requires a specific tab-delineated text file format to track samples spotted from plates onto microarray substrates; therefore, it was necessary to design additional database processes to reformat the WinPREP data. The resulting CloneTracker files were used with the VIRTEK SDDC-2 software to complete tracking between the parent cDNA clones and the individual microarray spots. CloneTracker subsequently exported a positional text file used by the companion IMAGENE image analysis software (BioDiscovery) after scanning.

\section{Microarray Production and Analysis}

The procedures described in Hegde et al. (6) were used to prepare and hybridize cDNA arrays using the VIRTEK spotter. Arrays were produced onto coated slides, dried, postprocessed, and stored in a desiccator until use. Labeled cRNA hybridization targets were produced (6) using SuperScript $\mathrm{II}^{\mathrm{TM}}$ reverse transcriptase (Invitrogen, Carlsbad, CA, USA) to incorporate Cyanine 3-dUTP and Cyanine 5-dUTP fluorophores (PerkinElmer Life Sciences) into 25-80 $\mu \mathrm{g}$ total RNA isolated from mouse kidney or liver using a bead milling process for tissue disruption (P.R. Hoyt and M.J. Doktycz, unpublished data and Reference 3). Hybridized arrays were imaged using the ScanArray at 5- or $10-\mu \mathrm{m}$ resolution.

\section{RESULTS AND DISCUSSION}

Probe preparation for DNA microarrays typically involves multiple sample preparation procedures. As shown in Figure 1 , these steps include plasmid preparation, PCR amplification, PCR cleanup, and PCR product characterization. While some steps may need to be conducted only initially, or perhaps on a yearly basis, they nonetheless represent an initial bottleneck for the construction of a high-density DNA microarray - especially considering the large number of reactions needed. Automation is a prerequisite for increased throughput and is highly desirable to reduce sample-handling errors. To automate microarray sample preparation and tracking with a minimum investment in equipment and space, we exploited the capabilities of the MultiPROBE II workstation and its WinPREP software.

Our first step in microarray probe preparation was plasmid DNA isolation. We found a better PCR success rate (approximately 97\%) using purified plasmid DNA than when direct amplification of bacterial lysates was used (data not shown). Many commercial kits are designed for high-throughput plasmid DNA purification, usually in 96-well format, and several are amenable to automation by liquid handling robots $(2,9,10,14)$. We chose the well-characterized QIAprep Turbo miniprep system $(8,11)$, although other plasmid kits are also suitable. This plasmid isolation kit requires a vacuum manifold to facilitate sample binding, washing, and elution from the purification resin. To eliminate sample cross-contamination during vacuum processing, we devised a custom, flowthrough plate support that precisely positioned the elution plate below the filter plate columns within the manifold base. During vacuum steps, each column nozzle was drawn into a microplate well upon compression of the manifold gasket. This directed the eluant sample into its collection well, effectively shielding it from adjacent wells, and cross-contamination was not observed after PCR or hybridization steps.

Figure 2A shows the gel electrophoresis results for a representative set of 192 plasmid preparations. Predominately covalently closed, supercoiled plasmid DNA was observed. Variations in DNA yields (e.g., upper versus lower rows) were largely due to intrinsic differences in plasmid amplification or culture growth and were consistently observed in replicate experiments. Typical yields were 5-30 $\mu \mathrm{g}$ DNA in a final volume of 100-150 $\mu \mathrm{L}$, as determined by fluorescence quantitation. Plasmid quality was assessed further by

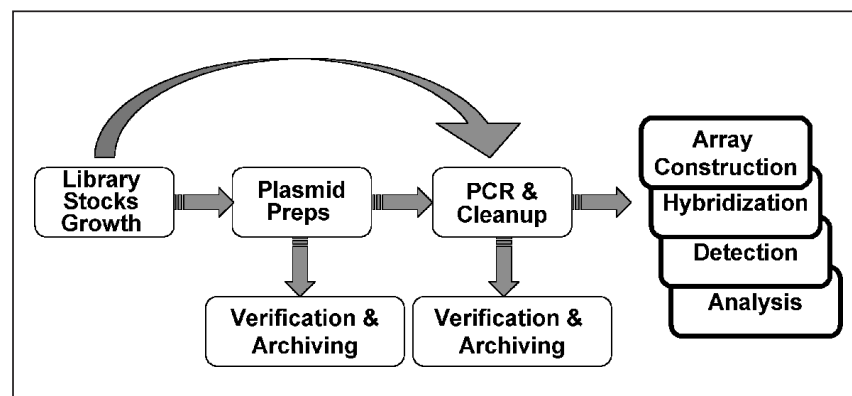

Figure 1. Flowchart diagram of the processing required for cDNA microarray production and analysis. 
Table 1. Typical Results for Throughput Times and Sample Numbers Processed

\begin{tabular}{|lcc|}
\hline Procedure & $\begin{array}{c}\text { No. Samples } \\
\text { Processed }\end{array}$ & $\begin{array}{c}\text { Time for } \\
\text { Procedurea (min) }^{\text {P }}\end{array}$ \\
\hline Plasmid Isolation & 192 & 114 \\
PCR Reaction Setup & 384 & 20 \\
PCR Cleanup & 192 & 54 \\
Gel Loading & 192 & 45 \\
DNA Quantitation & 192 & 72 \\
Redistributionb & 384 & 60 \\
aAfter instrument setup & & \\
bFour replicate 384-well plates prepared & \\
\hline
\end{tabular}

$\mathrm{A}_{260} / \mathrm{A}_{280}$ ratios and ultimately by $\mathrm{PCR}$. The average $\mathrm{A}_{260} / \mathrm{A}_{280}$ ratio was 1.7. Plasmid yields were also determined using absorbance measurements and found to be similar to values determined using fluorescence (data not shown). The average product length for the plates used was approximately $1 \mathrm{~kb}$, which is slightly less than the reported average insert length for the entire 15000 -clone set $(1.5 \mathrm{~kb})$ reported by Tanaka et al. (17).

The PCR cleanup protocol used liquid handling and vacuum steps similar to the plasmid isolation protocol. With the dual manifold setup and the QIAquick 96 Purification Kit, 192 PCR products were purified in under an hour. Typical PCR product yields range from 1 to $20 \mu \mathrm{g}$ for a $50-\mu \mathrm{L}$ reaction. Figure $2 \mathrm{~B}$ displays the electrophoresis results of 192 amplification reactions for the plasmid templates shown in Figure 2A. The results indicate that 187 of $192(97 \%)$ samples performed suitably as PCR templates and eight (4\%) showed multiple bands. Reactions producing multiple bands were consistent with other reports (6). Gel data indicated that PCR products were recovered with insignificant sample loss (data not shown).

Table 1 summarizes some typical results for throughput times and sample numbers processed. Clearly, up to 1000 clones can be prepared from bacterial stocks in less than one week's time using a single instrument, operated by a single researcher.

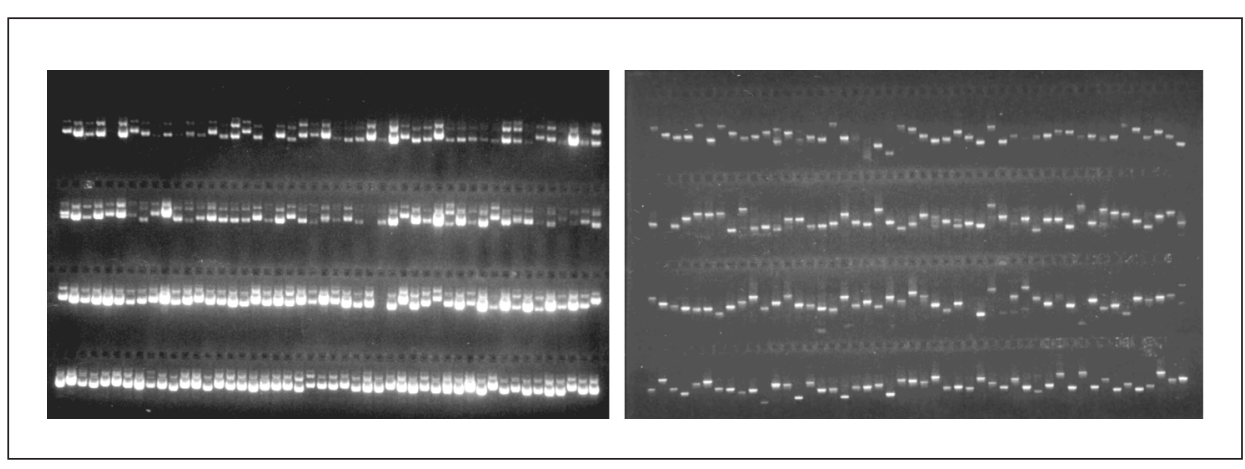

Figure 2. Ethidium bromide-stained agarose gel analysis of 192 samples automatically processed using the MultiPROBE workstation. Clones were from plates H3002 and H3074 of the Tanaka et al. clone set (17). (A) Left, plasmid DNA preparations ( $5 \mu \mathrm{L}$ of a $100-\mu \mathrm{L}$ eluant/lane). (B) Right, purified PCR products $(3 \mu \mathrm{L}$ of a $100-\mu \mathrm{L}$ eluant/lane).
One constraint for sample throughput is the need for effective quality-control procedures that match the probe production rate (i.e., non-rate limiting). Electrophoretic analysis addresses both DNA quality and quantity. We automated the process of loading samples onto electrophoresis gels using the MultiPROBE II to eliminate the tedium and reduce errors, while allowing users to attend to other functions. MultiPROBE sample loading is not faster than manual multichannel pipet loading but has the advantage of loading samples consecutively rather than using alternating well patterns.

Another quality check carried out by the MultiPROBE II is fluorescence-based DNA quantitation in 96-well format. Both plasmid DNA and PCR product yields were measured using PicoGreen and a fluorescence plate reader $(1,13)$. The advantages of this assay are its wide dynamic measurement range, use of standard low-cost plates, and its specificity for dsDNA. By accurately quantitating DNA before spotting, concentrations can be standardized, leading to consistent spot patterns. Quantitation assay setup and analysis of 96 samples were completed in $40 \mathrm{~min}$. The average DNA yield after PCR cleanup was $289 \mathrm{ng} / \mathrm{mL}$ ( $\mathrm{SD} \pm 87 \mathrm{ng} / \mathrm{mL}$ ). This coincides well with, and is complementary to, agarose gel results. Electrophoretic analysis is required to assess the presence of multiple products within a single reaction.

Sample tracking is obligated by the handling of large numbers of probes through multiple processes. A complication is the change from 96-well microplates (storage and purification) to 384-well format (chip spotting) and then further to pin-based spotting microarray patterns. We have automated the tracking of sample location using the MultiPROBE's Microsoft Access database to link liquid handling steps relative to plate names and clone well positions. Starting with clone descriptions on tab-delineated or Excel spreadsheets, database processes were devised to generate files that track clone position. We also moved the information into a commercial software package, CloneTracker, to follow the samples during microarray spotting and analysis.

Finally, we validated the DNA microarray production methods using gene expression analysis. Figure 3 displays section of hybridized cDNA array showing 352 unique cDNA probes, spotted in triplicate. These images show the changes in gene expression observed when hybridized to mouse liver and kidney using $192 \mathrm{cDNAs}$ prepared as described in this report. A known set of liver-specific genes was included in this experiment and is easily seen as bright green spots, verifying that the hybridization conditions were satisfactory and that our probes performed as expected when compared with probes with known responses. These arrays show excellent signal-to-noise ratio and demonstrate that the final PCR product binds well to the slide substrate and hybridizes efficiently to the labeled target. 


\section{MicROARRAY Technologies}

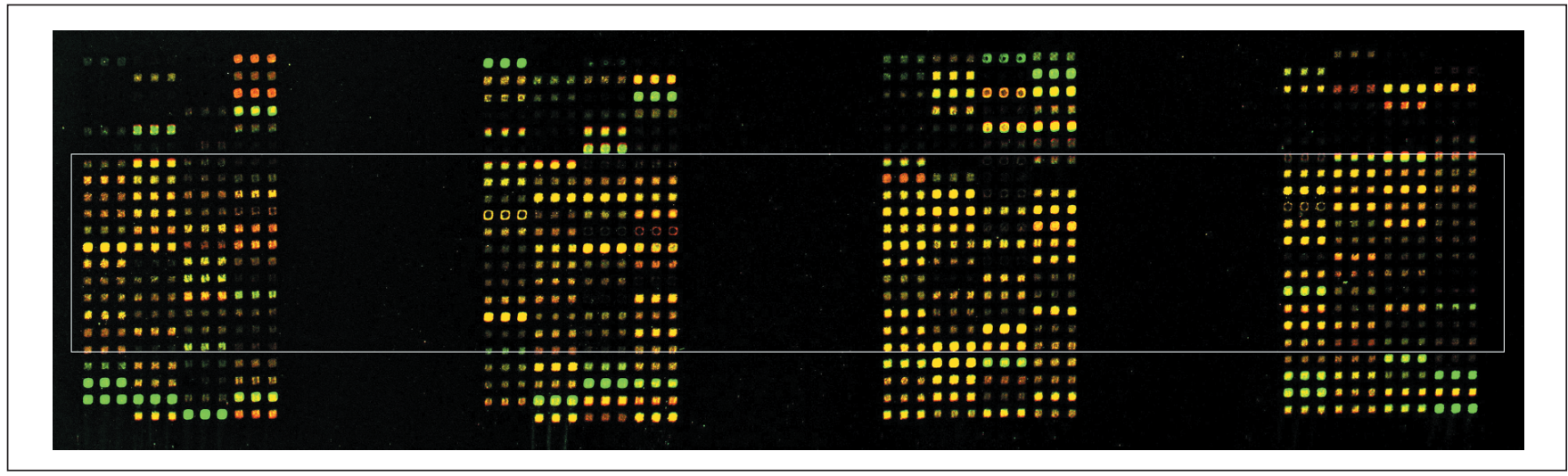

Figure 3. DNA differential expression microarray experiment using mouse kidney and mouse liver RNA targets. The box drawn around 192 spots in the middle of the array represents the same DNA samples shown in Figure 2. All DNA probes were produced as described in this paper. In this experiment, hybridization to liver target RNA is shown in green, and hybridization to kidney target RNA is shown in red.

\section{CONCLUSION}

High-density cDNA microarrays represent a powerful technology for defining gene expression but require the conversion of thousands of unique cDNA clones into DNA probes suit- able for spotting. As the expense of robotic equipment can be prohibitive, we wanted a multipurpose liquid handling system that would allow automation of many steps, thus maximizing the economy of our sample preparation process. The system chosen was Packard's Nucleic Acid Purification Workstation 
(16), which consists of the MultiPROBE II HT EX with Gripper Integration Platform, PVM Vacuum Manifold, DPC Shaker, and custom WinPREP Nucleic Acid Extraction template (15). This workstation was capable of carrying out fully automated vacuum filtration and other functions required for DNA probe processing in 96- and 384-well formats. This included plasmid DNA purification, PCR setup and cleanup, gel loading, fluorescent DNA quantification, and sample redistribution protocols. Workstation functions include pipetting, gripper (filter and collection plate relocation, manifold assembly-disassembly steps), shaking, timer control, and a dual-control vacuum system. Flexible accessory-mapping software facilitated the implementation of new labware/hardware. Tracking of sample order was enabled via WinPREP and Microsoft Access database. Custom queries and macros were implemented to automate generation of text files required for "downstream" microarray database analysis. New automated workstation protocols using the workstation greatly reduced user intervention and associated errors while increasing throughput. In summary, we developed a flexible platform for both high- and medium-throughput generation of probes suitable for high-density array production and analysis.

Further description of our automated microarray processing system (downloadable operation files, detailed protocols, descriptions of custom hardware, and process information) is available online at: http://HOMER.HSR.ORNL.GOV/CBPS/ Arraytechnology/arrayindex.html.

\section{ACKNOWLEDGMENTS}

We thank Ed Michaud, Jay Snoddy, Denise Schmoyer, and Christine Schar (Oak Ridge National Laboratory). This research was sponsored by the Laboratory Directed Research and Development Program of Oak Ridge National Laboratory, managed by UT-Battelle, LLC for the U. S. Department of Energy under contract no. DE-AC05-00OR22725, and support from Perkin-Elmer Life Sciences. This manuscript has been authored by a contractor of the U.S. government under contract no. DE-AC05-00OR22725. Accordingly, the U.S. government retains a nonexclusive, royalty-free license to publish or reproduce the published form of this contribution, or allow others to do so, for U.S. government purposes.

\section{REFERENCES}

1.Ahn, S., J. Costa, and J. Emanuel. 1996. PicoGreen quantitation of DNA: effective evaluation of samples pre- or post-PCR. Nucleic Acids Res. 24:2623-2625.

2.Beg, O.U. and R.G. Holt. 1998. A cost-effective plasmid purification protocol suitable for fluorescent automated DNA sequencing. Mol. Biotechnol. 9:79-83

3.Chomczynski, P. and N. Sacchi. 1987. Single-step method of RNA isolation by acid guanidinium thiocyanate-phenol-chloroform extraction. Anal. Biochem. 162:156-159.

4.Engelstein, M., T. Aldredge, D. Madan, J. Smith, J. Mao, D. Smith, and P. Rice. 1998. An efficient, automatable template preparation for high throughput sequencing. Microb. Comp. Genomics 3:237-241.

5.Garner, H., B. Armstrong, and D. Kramarsky. 1992. High-throughput DNA preparation system. Genet. Anal. Tech. Appl. 9:134-139.

6.Hegde, P., R. Qi, K. Abernathy, C. Gay, S. Dharap, R. Gaspard, J.
Earle-Hughes, E. Snesrud, et al. 2000. A concise guide to cDNA microarray analysis. BioTechniques 29:548-562.

7.Huang, G., K. Wang, C. Kuo, B. Paeper, and L. Hood. 1994. A highthroughput plasmid DNA preparation method. Anal. Biochem. 223:3538.

8.Kirschner, L. and C. Stratakis. 1999. Large-scale preparation of sequence-ready bacterial artificial chromosome DNA using QIAGEN columns. BioTechniques 27:72-74.

9.Konecki, D. and J. Phillips. 1998. TurboPrep II: an inexpensive, highthroughput plasmid template preparation protocol. BioTechniques 24:286293.

10.Neudecker, F. and S. Grimm. 2000. High-throughput method for isolating plasmid DNA with reduced lipopolysaccharide content. BioTechniques 28:107-109.

11.Pogue, R., M. Cook, L. Livingstone, and S. Hunt. 1993. Preparation of template for automated sequencing using QIAGEN resin. BioTechniques 15:376-381.

12.Sambrook, J., E. Fritsch, and T. Maniatis. 1989. Molecular Cloning: A Laboratory Manual, 2nd ed. CSH Laboratory Press, Cold Spring Harbor, NY.

13.Singer, V., L. Jones, S. Yue, and R. Haugland. 1997. Characterization of PicoGreen reagent and development of a fluorescence-based solution assay for double-stranded DNA quantitation. Anal. Biochem. 249:228 238.

14.Skowronski, E.W., N. Armstrong, G. Andersen, M. Macht, and P.M. McCready. 2000. Magnetic, microplate-format plasmid isolation protocol for high-yield, sequencing-grade DNA. BioTechniques 29:786-792.

15.Tack, L., J. Van Dinther, and P. Ahrweiler. 2001. Fully automated nucleic acid extraction using the MultiPROBE II EX with Gripper Integration Platform and PVM Vacuum Filtration System. Packard BioScience Liquid Handling Application Note LHA-017:1-8. Packard Bioscience, Downers Grove, IL.

16. Tack, L. and J. Van Dinther. 2002. Automated Nucleic Acid Purification Workstation. Packard BioScience Liquid Handling Application Note LHA-023:1-8. Packard Bioscience, Downers Grove, IL.

17.Tanaka, T.S., A. Jaradat, M.K. Lim, G.J. Kargul, X. Wang, M.J. Grahovac, S. Pantano, Y. Sano, et al. 2000. Genome-wide expression profiling of mid-gestation placenta and embryo using a 15,000 mouse developmental cDNA microarray. Proc. Natl. Acad. Sci. USA 97:9127-9132.

Address correspondence to:

Dr. Peter R. Hoyt

Life Sciences Division

Oak Ridge National Laboratory

P.O. Box 2008, MS 6123

Oak Ridge, TN 37831, USA

e-mail: hoytpr@ornl.gov

For reprints of this or any other article, contact Reprints@BioTechniques.com 